\title{
Topological rigidity of Hamiltonian loops and quantum homology
}

\author{
François Lalonde* \\ Université du Québec à Montréal \\ (flalonde@math.uqam.ca) \\ Dusa McDuff ${ }^{\dagger}$ \\ State University of New York at Stony Brook \\ (dusa@math.sunysb.edu) \\ Leonid Polterovich $\ddagger$ \\ Tel-Aviv University \\ (polterov@math.tau.ac.il) \\ October 1, 1997
}

\section{§1. Main result}

Let $G$ be a connected subgroup of the group $\operatorname{Diff}(M)$ of diffeomorphisms of a manifold $M$. It is well known that every element $\phi \in \pi_{1}(G$,id) defines an endomorphism $\partial_{\phi}: H_{*}(M, \mathbf{Q}) \rightarrow H_{*+1}(M, \mathbf{Q})$ as follows. Choose a loop $\left\{\phi_{t}\right\}, t \in S^{1}$, of diffeomorphisms from $G$ representing $\phi$ and a cycle $C$ in $M$. Then the homology class $\partial_{\phi}([C])$ is represented by the cycle $S^{1} \times C \rightarrow M$ which is spanned by $C$ under the loop $\left\{\phi_{t}\right\}$.

Suppose now that $(M, \omega)$ is a closed symplectic manifold, and take $G$ to be its group $\operatorname{Ham}(M, \omega)$ of Hamiltonian diffeomorphisms. In this paper, we discuss the following statement. As we will see in section 2 it has a number of applications to the geometry and topology of the group of symplectomorphisms.

Theorem 1.A. Let $\phi$ be a loop in the group $\operatorname{Ham}(M, \omega)$ of Hamiltonian diffeomorphisms. Then $\partial_{\phi}$ vanishes identically for all $\phi \in \pi_{1}(\operatorname{Ham}(M, \omega)$, id).

Below we give the proof of this statement when $M$ is 4-dimensional as well as for some higher dimensional symplectic manifolds - the so-called spherically

*Partially supported by NSERC grant OGP 0092913 and FCAR grant ER-1199.

$\dagger$ Partially supported by NSF grant DMS 9704825.

$\ddagger$ All three authors supported by a NSERC Collaborative Project Grant CPG0163730. 
monotone manifolds and other manifolds where $J$-holomorphic curves are wellbehaved. See $\S 6$ for the precise assumptions on $(M, \omega)$. We believe that all theorems stated in this paper do hold in the general case too, which forms the subject of our forthcoming paper [LMP2]; it requires the use of the GromovWitten invariants on arbitrary manifolds as developed by Fukaya-Ono [FO], Li-Tian [LiT], Liu-Tian [LT], Ruan [R], and Hofer-Salamon [HS], and involves a deeper and more technical analysis. There are still a number of details to be checked.

However, all the basic geometric ideas are already present in the particular case treated here. An early version of these ideas was described in the survey article by McDuff [M2].

These ideas were inspired by a recent result of Seidel [Se] who discovered a canonical action of a certain extension of the group $\pi_{1}(\operatorname{Ham}(M, \omega))$ on the quantum homology ring of $M$ that arises from the natural action of the element $\phi$ on the loop space of $M$. Seidel defines this action under the additional assumptions mentioned above, and we will show that in this case 1.A can be deduced from Seidel's result by simple geometric arguments.

Notice that the particular case of 1.A stating that the map

$$
\partial_{\phi}: H_{0}(M, \mathbf{Q}) \rightarrow H_{1}(M, \mathbf{Q})
$$

vanishes simply means that the orbits of a periodic Hamiltonian flow 1 are homologous to zero. This is a classical result and is very easy to prove (see [BP], II-1.3). Also when $\phi$ is a Hamiltonian circle action the statement of 1.A immediately follows from a result of Kirwan (see 3.C below.)

To our knowledge, the results of the present paper constitute the first application of Quantum homology to Hamiltonian mechanics.

The paper is organized as follows. In $\S 2$ we explain what "topological rigidity" is, and we derive from 1.A a new case of the Flux Conjecture. In $\S 3$ we reformulate 1.A in the more geometric language of symplectic fibrations over the 2-sphere. In $\S 4$ we describe Seidel's action and give the proof of 1.A. in the particular cases explained in $\S 6$. $\S 5$ contains a refinement of Seidel's theory. In particular we construct a representation of the group $\pi_{1}(\operatorname{Ham}(M, \omega))$ into the group of automorphisms of an extension of the usual quantum cohomology ring of $M$. Finally, in the last section, $\S 6$, we discuss the conditions on $M$ under which the results of the present paper hold, and what is therefore left to prove in [LMP2].

\section{§2. Rigidity of Hamiltonian loops}

Let $\operatorname{Symp}_{0}(M, \omega)$ be the connected component of the identity in the group of all symplectomorphisms of $(M, \omega)$. We will say that the class $\phi \in \pi_{1}(\operatorname{Diff}(M)$, id $)$

\footnotetext{
${ }^{1}$ In this note, when no specific mention is made, "Hamiltonian flow" is understood in the general sense of a time-dependent Hamiltonian.
} 
has an $\omega$-symplectic representative if it may be represented by a loop $\left\{\phi_{t}\right\}, t \in$ $S^{1}$, in $\operatorname{Symp}_{0}(M, \omega)$ based at the identity. The loop $\left\{\phi_{t}\right\}$ is $\omega$-Hamiltonian if it is the flow (with respect to $\omega$ ) of a time-dependent Hamiltonian function $H_{t}$. We are interested in the question of which classes in $\pi_{1}\left(\operatorname{Symp}_{0}(M, \omega)\right.$, id) have Hamiltonian representatives, or equivalently of when $\left\{\phi_{t}\right\}$ is homotopic (through $\omega$-symplectic loops) to a Hamiltonian loop.

Theorem 2.A. Suppose that $\omega_{1}$ and $\omega_{2}$ are two symplectic forms on $M$ and that $\phi \in \pi_{1}\left(\operatorname{Diff}(M)\right.$, id) contains $\omega_{i}$-symplectic representatives $\left\{\phi_{t}^{i}\right\}$, for $i=$ 1,2 . Then $\phi$ contains a $\omega_{1}$-Hamiltonian representative if and only if it contains a $\omega_{2}$-Hamiltonian representative.

In other words, once we know that a loop has a symplectic representative, the question of whether or not this representative can be chosen to be Hamiltonian is independent of the choice of the symplectic form, and in particular of its cohomology class. This forms the content of the phenomenon of topological rigidity of Hamiltonian loops which is announced in the title of the present note. 2

One situation in which this applies is when $\omega^{\prime}$ is a $C^{\infty}$-small perturbation of $\omega$ in the space of closed 2-forms. Using Moser's argument one can easily show that any given $\omega$-symplectic loop $\left\{\phi_{t}\right\}$ can be perturbed to an $\omega^{\prime}$-symplectic loop $\left\{\phi_{t}^{\prime}\right\}$ provided that $\omega^{\prime}$ is sufficiently $C^{\infty}$-close to $\omega$.

Corollary 2.B. Assume in the above situation that the loop $\left\{\phi_{t}\right\}$ is homotopic in $\operatorname{Symp}_{0}(M, \omega)$ to a Hamiltonian loop. Then the loop $\left\{\phi_{t}^{\prime}\right\}$ is homotopic in $\operatorname{Symp}_{0}\left(M, \omega^{\prime}\right)$ to a Hamiltonian loop.

In other words, the property of a loop of symplectomorphisms to be Hamiltonian up to homotopy is stable with respect to (small) deformations of the symplectic structure.

The above theorem is an almost immediate consequence of 1 .A because of the characterization of Hamiltonian loops via the flux homomorphism. Recall that the flux homomorphism

$$
F_{\omega}: \pi_{1}\left(\operatorname{Symp}_{0}(M, \omega), \mathrm{id}\right) \rightarrow H^{1}(M, \mathbf{R})
$$

can be defined as follows. For an element $\phi \in \pi_{1}\left(\operatorname{Symp}_{0}(M, \omega)\right.$,id $)$ and for a class $a \in H_{1}(M, \mathbf{Q})$ set

$$
\left(F_{\omega}(\phi), a\right)=\left([\omega], \partial_{\phi} a\right),
$$

where $\partial_{\phi}$ is the homomorphism defined in $\S 1$ and $(\cdot, \cdot)$ is the natural pairing. It is well-known that $\phi$ is represented by a Hamiltonian loop if and only if $F_{\omega}(\phi)=0$. (See Chapter 10 of [MS2], for example.)

${ }^{2}$ Actually, the proof of Theorem 2.A shows that the following stronger statement holds: if the class $\phi$ contains a $\omega_{1}$-Hamiltonian representative, then any $\omega_{2}$-symplectic loop is homotopic through $\omega_{2}$-symplectic loops to a $\omega_{2}$-Hamiltonian loop. This result implies the following slighly different version of 2.A: the loop $\left\{\phi_{t}^{1}\right\}$ is homotopic (through $\omega_{1}$-symplectic loops) to a Hamiltonian loop if and only if the same is true for $\left\{\phi_{t}^{2}\right\}$. 
Proof of 2.A: Observe that the homomorphism $\partial: H_{1}(M, \mathbf{Q}) \rightarrow H_{2}(M, \mathbf{Q})$ associated to the loop $\left\{\phi_{t}^{1}\right\}$ equals that associated to $\left\{\phi_{t}^{2}\right\}$, since the loops are homotopic in the group of diffeomorphisms. If we know that $\left\{\phi_{t}^{1}\right\}$ for example is homotopic to a Hamiltonian loop then 1.A applied to $\left\{\phi_{t}^{1}\right\}$ implies that $\partial$ vanishes. Thus the loop $\left\{\phi_{t}^{2}\right\}$ has zero flux and hence it is Hamiltonian up to homotopy.

Define the flux subgroup $\Gamma_{\omega} \in H^{1}(M, \mathbf{R})$ as the image of the flux homomorphism. The importance of this notion is due to the fact that $\operatorname{Ham}(M, \omega)$ is $C^{1}$-closed in $\operatorname{Symp}_{0}(M, \omega)$ if and only if $\Gamma_{\omega}$ is a discrete subgroup of $H^{1}(M, \mathbf{R})$. The statement that $\Gamma_{\omega}$ is discrete is known as the $C^{1}$-flux conjecture. It is proved in various cases with the use of both "soft" and "hard" methods, however it is still unsolved in full generality: see Lalonde-McDuff-Polterovich [LMP1]. The technique of the present paper allows it to be established in the following new case.

Theorem 2.C. The flux conjecture holds for all closed symplectic manifolds $\left(M^{2 n}, \omega\right)$ with first Betti number equal to 1.

Note that there are plenty of closed symplectic manifolds with first Betti number equal to 1 (see Gompf $[\mathrm{G}]$ ), though none of the interesting new examples are known to have nontrivial $\pi_{1}\left(\operatorname{Symp}_{0}(M)\right.$; id). Theorem 2.C follows immediately from the next more general statement.

Theorem 2.D. The rank over $\mathbf{Z}$ of the group $\pi_{1}\left(\operatorname{Symp}_{0}(M)\right) / \pi_{1}(\operatorname{Ham}(M))$ (which is identified with $\Gamma_{\omega}$ by the Flux homomorphism) is not greater than the first Betti number of $M$. In particular, it is finitely generated over $\mathbf{Z}$.

Proof: If the first statement does not hold, there are symplectic loops $\phi_{1}, \ldots, \phi_{m}$ with $m>\beta_{1}(M)$ whose fluxes $\lambda_{i}=F_{\omega}\left(\phi_{i}\right)$ are independent over $\mathbf{Z}$ in $H^{1}(M, \mathbf{R})$. Perturb the form $\omega$ to a rational form $\omega^{\prime}$ and then perturb the loops $\phi_{i}$ to $\omega^{\prime}$ symplectic loops $\phi_{i}^{\prime}$ with fluxes $\lambda_{i}^{\prime}$. Since the $\lambda_{i}^{\prime}$ are rational, there is a non-trivial integral linear combination of them $\lambda^{\prime}=\Sigma_{i} n_{i} \lambda_{i}^{\prime}$ that vanishes. Therefore by Theorem 1.A the homomorphism $\partial_{\phi^{\prime}}$ associated to the loop $\phi^{\prime}=\Pi_{i}\left(\phi_{i}^{\prime}\right)^{n_{i}}$ vanishes. Thus $\partial_{\phi}=0$ for the loop $\phi=\Pi_{i}\left(\phi_{i}\right)^{n_{i}}$ and hence this loop has zero flux. But this means that $\phi$ is in $\pi_{1}(\operatorname{Ham}(M))$, contradicting the hypothesis.

\section{$\S 3$. Symplectic fibrations over $S^{2}$}

There is a correspondence between loops in the group of symplectic diffeomorphisms and symplectic fibrations over $S^{2}$ with fiber $(M, \omega)$. By definition a symplectic fibration is a fibration such that the changes of trivialisation preserve a given symplectic form $\omega$ on the fibers. In other words, the structure group of the fibration is $\operatorname{Symp}(M)$. The correspondence is given by assigning to each symplectic loop $\phi_{t \in[0,1]}$ in $\operatorname{Symp}_{0}(M)$ the fibration $(M, \omega) \rightarrow P_{\phi} \rightarrow S^{2}$ obtained 
by gluing a copy of $D_{2}^{+} \times M$ with another $D_{2}^{-} \times M$ along their boundary in the following way:

$$
(2 \pi t, x) \mapsto\left(-2 \pi t, \phi_{t}(x)\right) .
$$

(Here $D_{2}$ is the closed disc of radius 1 of the plane.) In what follows we always assume that the base $S^{2}$ is oriented, and with orientation induced from $D_{2}^{+}$. Note that this correspondence can be reversed: given a symplectic fibration over the oriented 2-sphere together with an identification of one fiber with $M$, one can reconstruct the homotopy class of $\phi$.

An important topological tool for the study of such fibrations is the Wang exact sequence:

$$
\ldots \rightarrow H_{j-1}(M, \mathbf{Z}) \stackrel{\partial_{\phi}}{\rightarrow} H_{j}(M, \mathbf{Z}) \stackrel{i}{\rightarrow} H_{j}\left(P_{\phi}, \mathbf{Z}\right) \stackrel{\cap[M]}{\rightarrow} H_{j-2}(M, \mathbf{Z}) \rightarrow \ldots
$$

This sequence can be easily derived from the exact sequence of the pair $\left(P_{\phi}, M\right)$, where $M$ is identified with a fiber of $P_{\phi}$. The important point for us is, of course, that the boundary map $H_{j-1}(M) \rightarrow H_{j}(M)$ is precisely the homomorphism $\partial_{\phi}$ that interests us. Thus $\partial_{\phi}$ vanishes exactly when the inclusion $i$ is injective or, equivalently, when the restriction map $\cap[M]$ is surjective.

We say that a symplectic fibration is Hamiltonian if the corresponding loop of symplectomorphisms is homotopic to a Hamiltonian loop. The crucial point is that $P_{\phi}$ is Hamiltonian if and only if the cohomology class of the symplectic structure on the fiber extends to a cohomology class on the total space. This is most easily seen if one considers the Wang sequence on cohomology

$$
\ldots \rightarrow H^{j+1}\left(P_{\phi}\right) \stackrel{\text { restr }}{\rightarrow} H^{j+1}(M) \stackrel{\partial_{\phi}^{*}}{\rightarrow} H^{j}(M) \rightarrow H^{j+2}\left(P_{\phi}\right) \rightarrow \ldots
$$

where $\partial_{\phi}^{*}$ denotes the dual of $\partial_{\phi}$, and notes that $\phi$ is Hamiltonian exactly when $\partial_{\phi}^{*}([\omega])=0$.

With this language, Theorem 1.A above is equivalent to the following statement.

Theorem 3.A. Let $\phi$ be a Hamiltonian loop on a closed symplectic manifold $(M, \omega)$. Then the homomorphism $i: H_{*}(M, \mathbf{Q}) \rightarrow H_{*}\left(P_{\phi}, \mathbf{Q}\right)$ is injective.

The proof of this statement is sketched in the next section. The formulation of the rigidity phenomenon 2.A in the language of symplectic fibrations is especially simple.

Theorem 3.B. Let $\phi$ be a Hamiltonian loop on a closed symplectic manifold $(M, \omega)$. Consider the connected component of $P_{\phi}$ in the space of all symplectic fibrations with fiber $M$ and base $S^{2}$ (where the symplectic form on $M$ is allowed to vary). Then the whole connected component is formed of Hamiltonian fibrations. 
Remark 3.C. Certain special cases of 3.A and 3.B are already known. One of them was pointed out to us by Seidel, namely when the structure of the symplectic fibration $p: P_{\phi} \rightarrow S^{2}$ comes from a Kähler structure on the total space $P_{\phi}$ such that the projection $p$ is holomorphic. In this situation 3.A and 3.B follow from a result due to Deligne which states that the Leray spectral sequence of $P_{\phi}$ degenerates: see Chapter 3.5 in Griffiths-Harris [GH]. Another special case is when $\phi$ is generated by a circle action. In this case, one considers the equivariant cohomology $H_{S^{1}}^{*}(M, \mathbf{Q})$ that is defined to be the usual cohomology of the homotopy quotient

$$
M / / S^{1}=E S^{1} \times_{S^{1}} M,
$$

where $\pi: E S^{1} \rightarrow B S^{1}=\mathbf{C} P^{\infty}$ is the universal $S^{1}$-bundle: see Kirwan [K]. It is easy to check that the bundle $P_{\phi} \rightarrow S^{2}$ is just the restriction of the bundle $M / / S^{1} \rightarrow \mathbf{C} P^{\infty}$ to $\mathbf{C} P^{1}$. Further, one can check that the vanishing of $\partial_{\phi}$ is equivalent to the degeneration of the spectral sequence for the cohomology of $M / / S^{1}$, a fact that is proved by Kirwan in $[\mathrm{K}]$ by using localization formulas. Thus 3.A gives an alternative proof of this degeneration.

Since $p: P_{\phi} \rightarrow S^{2}$ is a Hamiltonian fibration it carries a natural deformation class of symplectic forms given by the weak coupling construction. Recall that the coupling class $u_{\phi} \in H^{2}\left(P_{\phi}, \mathbf{R}\right)$ is the (unique) class whose top power vanishes, and whose restriction to a fiber coincides with the cohomology class of the fiberwise symplectic structure. Let $\tau$ be a positive generator of $H^{2}\left(S^{2}, \mathbf{Z}\right)$. The deformation class above consists of symplectic forms $\Omega$ which represent the cohomology class of the form $u_{\phi}+\kappa p^{*} \tau(\kappa>>0)$ and extend the fiberwise symplectic structure. It is always possible to choose $\Omega$ so that it is a product with respect to the given product structure near the fibers $M_{0}$ at $0 \in D_{2}^{+}$and $M_{\infty}$ at $0 \in D_{2}^{-}$: see the proof of Lemma 3.E below.

Besides the coupling class $u_{\phi}$, the total space $P_{\phi}$ carries another canonical second cohomology class

$$
c_{\phi}=c_{1}\left(T P_{\phi}^{\mathrm{vert}}\right) \in H^{2}\left(P_{\phi}, \mathbf{R}\right)
$$

that is defined to be the first Chern class of the vertical tangent bundle.

Remark 3.D. The existence of this extension $c_{\phi}$ of the first Chern class $c_{1}(T M)$ provides a natural explanation of a phenomena that was first observed by McDuff in [M1] and rediscovered by Lupton-Oprea [LO], namely that the flux homomorphism $F_{\omega}: \pi_{1}\left(\operatorname{Symp}_{0}(M, \omega)\right) \rightarrow H^{1}(M, \mathbf{R})$ vanishes when the symplectic class $[\omega]$ is a multiple of $c_{1}$.

Both classes $u_{\phi}, c_{\phi}$ behave well under compositions of loops. More precisely, consider two elements $\phi, \psi \in \pi_{1}(\operatorname{Ham}(M, \omega))$ and their composite $\psi * \phi$. This can be represented either by the product $\psi_{t} \circ \phi_{t}$ or by the concatenation of loops. It is not hard to check that the bundle $P_{\psi * \phi}$ can be realised as the fiber 
sum $P_{\psi} \# P_{\phi}$ obtained as follows. Let $M_{\phi, \infty}$ denote the fiber at $0 \in D_{2}^{-}$in $P_{\phi}$ and $M_{\psi, 0}$ the fiber at $0 \in D_{2}^{+}$in $P_{\psi}$. Cut out open product neighborhoods of each of these fibers and then glue the complements by an orientation reversing symplectomorphism of the boundary. The resulting space may be realised as

$$
D_{2}^{+} \times M \cup_{\alpha_{\phi,-1}} S^{1} \times[-1,1] \cup_{\alpha_{\psi, 1}} D_{2}^{-} \times M,
$$

where

$$
\alpha_{\phi,-1}(2 \pi t, x)=\left(2 \pi t,-1, \phi_{t}(x)\right), \quad \alpha_{\psi, 1}\left(2 \pi t, 1, \psi_{t}(x)\right)=(2 \pi t, x),
$$

and this may clearly be identified with $P_{\psi * \phi}$. Set

$$
V_{\phi}=D_{2}^{+} \times M \cup S^{1} \times[-1,1 / 2), \quad V_{\psi}=S^{1} \times(-1 / 2,1] \cup D_{2}^{-} \times M .
$$

The next lemma follows imediately from the construction of the coupling form via symplectic connections: see [P2] or [MS2].

Lemma 3.E. The classes $u_{\psi * \phi}$ and $c_{\psi * \phi}$ are compatible with the decomposition $P_{\psi * \phi}=V_{\psi} \cup V_{\phi}$ in the sense that their restrictions to $V_{\psi} \cap V_{\phi}=(-1 / 2,1 / 2) \times$ $S^{1} \times M$ equal the pullbacks of $[\omega]$ and $c_{1}(T M)$.

Corollary 3.F. For every $k \in\{1, \ldots, n\}$ the map

$$
\phi \mapsto \int_{P_{\phi}}\left(c_{\phi}\right)^{k}\left(u_{\phi}\right)^{n+1-k}
$$

defines a homomorphism $I_{k}: \pi_{1}(\operatorname{Ham}(M, \omega)) \rightarrow \mathbf{R}$.

Remark 3.G. When $(M, \omega)$ is monotone 3 the homomorphism $I_{1}$ agrees with the mixed action-Maslov homomorphism $I$ defined by Polterovich in [P1]. However, although they are both defined in the spherically monotone case, they can differ since $I$ depends only on the values of $c_{\phi}$ on spheres, while $I_{1}$ may not. Indeed

$$
I_{1}(\phi)=c_{\phi}\left(\mathrm{PD}\left(\left(u_{\phi}\right)^{n}\right),\right.
$$

and the Poincaré dual $\operatorname{PD}\left(\left(u_{\phi}\right)^{n}\right) \in H_{2}\left(P_{\phi}\right)$ need not be in $H_{2}^{S}(M) \otimes \mathbf{R}$. For example, if $M$ is a nontrivial $S^{2}$-bundle over a Riemann surface of genus $>0$ and $\phi$ is given by an $S^{1}$-action that rotates the fibers of $M$ it is not hard to check that $\operatorname{PD}\left(\left(u_{\phi}\right)^{2}\right)$ is not spherical.

\section{$\S 4$ Seidel's maps $\Psi_{\phi, \sigma}$}

We start with the definition of the quantum cohomology ring of $M$. In view of our purposes in the next section, we will give two versions of this definition,

\footnotetext{
${ }^{3}$ In this note we will say that $(M, \omega)$ is monotone if, for some positive $\kappa \in \mathbf{R}, c_{1}(T M)=$ $\kappa[\omega]$ on the whole of $H_{2}(M)$. If this equation holds only on the spherical part $H_{2}^{S}(M)$ of $H_{2}$ we will call $(M, \omega)$ spherically monotone.
} 
one with rational and one with real coefficients. To simplify our formulas we will denote the first Chern class $c_{1}(T M)$ of $M$ by $c$.

Let $\Lambda$ be the usual (rational) Novikov ring of the group $\mathcal{H}=H_{2}^{S}(M, \mathbf{Z}) / \sim$ with valuation $\omega($.$) where B \sim B^{\prime}$ if $\omega\left(B-B^{\prime}\right)=c\left(B-B^{\prime}\right)=0$, and let $\Lambda_{R}$ be the analogous (real) Novikov ring based on the group $\mathcal{H}_{R}=H_{2}^{S}(M, \mathbf{R}) / \sim$. Thus the elements of $\Lambda$ have the form

$$
\sum_{B \in \mathcal{H}} \lambda_{B} e^{B}
$$

where for each $\kappa$ there are only finitely many nonzero $\lambda_{B} \in \mathbf{Q}$ with $\omega(B)<\kappa$, and the elements of $\Lambda_{R}$ are

$$
\sum_{B \in \mathcal{H}_{R}} \lambda_{B} e^{B}
$$

where $\lambda_{B} \in \mathbf{R}$ and there is a similar finiteness condition. f Set $Q H_{*}(M)=$ $H_{*}(M) \otimes \Lambda$ and $Q H_{*}\left(M, \Lambda_{R}\right)=H_{*}(M) \otimes \Lambda_{R}$. Then $Q H_{*}(M)$ is $\mathbf{Z}$-graded with $\operatorname{deg}\left(a \otimes e^{B}\right)=\operatorname{deg}(a)-2 c(B)$. It is best to think of $Q H_{*}\left(M, \Lambda_{R}\right)$ as $\mathbf{Z} / 2 \mathbf{Z}$-graded with

$$
Q H_{\mathrm{ev}}=H_{\mathrm{ev}}(M) \otimes \Lambda_{R}, \quad Q H_{\text {odd }}=H_{\text {odd }}(M) \otimes \Lambda_{R} .
$$

With respect to the quantum intersection product (defined in $\S 5$ below) both versions of quantum homology are graded-commutative rings with unit $[M]$. Further, the units in $Q H_{\mathrm{ev}}\left(M, \Lambda_{R}\right)$ form a group $Q H_{\mathrm{ev}}\left(M, \Lambda_{R}\right)^{\times}$that acts on $Q H_{*}\left(M, \Lambda_{R}\right)$ by quantum multiplication.

Now we describe how Seidel arrives at an action of the loop $\phi$ on the quantum homology of $M$. Denote by $\mathcal{L}$ the space of contractible loops in the manifold $M$. Fix a constant loop $x_{*} \in \mathcal{L}$, and define a covering $\widetilde{\mathcal{L}}$ of $\mathcal{L}$ with the base point $x_{*}$ as follows. Note first that a path between $x_{*}$ and a given loop $x$ can be considered as a 2-disc $u$ in $M$ bounded by $x$. Then the covering $\tilde{\mathcal{L}}$ is defined by saying that two paths are equivalent if the 2 -sphere $S$ obtained by gluing the corresponding discs has $\omega(S)=c(S)=0$. Thus the covering group of $\widetilde{\mathcal{L}}$ coincides with the abelian group $\mathcal{H}$.

Let $\phi=\left\{\phi_{t}\right\}$ be a loop of Hamiltonian diffeomorphisms. Because the orbits $\phi_{t}(x), t \in[0,1]$, of $\phi$ are contractible (see [LMP1]), one can define a mapping $T_{\phi}: \mathcal{L} \rightarrow \mathcal{L}$ which takes the loop $\{x(t)\}$ to a new loop $\left\{\phi_{t} x(t)\right\}$. Let $\widetilde{T}_{\phi}$ be a lift of $T_{\phi}$ to $\tilde{\mathcal{L}}$. To choose such a lift one should specify a homotopy class of paths in $\mathcal{L}$ between the constant loop and an orbit of $\left\{\phi_{t}\right\}$. It is not hard to see that in the language of symplectic fibrations this choice of lift corresponds to a choice of an equivalence class $\sigma$ of sections of $P_{\phi}$, where two sections are identified if their values under $c_{\phi}$ and $u_{\phi}$ are equal. Thus the lift can be labelled $\widetilde{T}_{\phi, \sigma}$.

\footnotetext{
${ }^{4}$ In [Se] Seidel works with a simplified version of the Novikov ring $\Lambda$ where the coefficients $\lambda_{B}$ affecting $e^{B}, B \in \mathcal{H}$, are elements of $\mathbf{Z} / 2 \mathbf{Z}$. However, his results extend in a staightforward way to the case of rational coefficients by taking into account orientations on the moduli spaces of pseudo-holomorphic curves. Let us emphasize that in our definition of $\Lambda_{R}$ not only the coefficients $\lambda_{B}$ are real, but also the exponents $B$ belong to a real vector space $\mathcal{H}_{R}$.
} 
Recall now that the Floer homology $H F_{*}(M)$ can be considered as the Novikov homology of the action functional on $\widetilde{\mathcal{L}}$. Therefore $\widetilde{T}_{\phi, \sigma}$ defines a natural automorphism $\left(\widetilde{T}_{\phi, \sigma}\right)_{*}$ of $H F_{*}(M)$. Further, if $\Phi: H F_{*}(M) \rightarrow H Q_{*}(M)$ is the canonical isomorphism constructed in Piunikhin-Salamon-Schwartz [PSS], there is a corresponding automorphism $\Psi_{\phi, \sigma}$ of $Q H_{*}(M)$ given by

$$
\Psi_{\phi, \sigma}=\Phi \circ\left(\widetilde{T}_{\phi, \sigma}\right)_{*} \circ \Phi^{-1} .
$$

This gives rise to an action of the group of all pairs $(\phi, \sigma)$ on $Q H_{*}(M)$.

Seidel shows that when $M$ satisfies a suitable semi-positivity condition the map $\Psi_{\phi, \sigma}: Q H_{*}(M) \rightarrow Q H_{*}(M)$ is in fact induced by quantum multipication by an element of $Q H_{\mathrm{ev}}(M)^{\times}$that is formed from the moduli space of all $J$ holomorphic sections of $P_{\phi}$. In our work we in a sense go backwards. We give a new definition of the maps $\Psi_{\phi, \sigma}$ that does not explicitly mention Floer homology and will prove that they are isomorphisms by a direct gluing argument. We will see in the next section that our map does agree with Seidel's. Further, our version of the definition no longer restricts us to using the coefficients $\Lambda$ via the covering $\widetilde{\mathcal{L}} \rightarrow \mathcal{L}$. Instead we will consider the extension $\Lambda_{R}$, which will allow us to define an action of the group $\pi_{1}$ (Ham) itself.

Let $\Omega$ be a symplectic form on $P_{\phi}$ that extends $\omega$ and is in the natural deformation class $u_{\phi}+\kappa p^{*}(\tau)$. As above, define an equivalence relation on the set of homology classes of sections of $P_{\phi}$ by identifying two such classes if their values under $c_{\phi}$ and $u_{\phi}$ are equal. Then, given a loop of Hamiltonian diffeomorphisms $\phi$ on $M$, and an equivalence class of sections $\sigma$ of $P_{\phi}$ with $d=2 c_{\phi}(\sigma)$, define a $\Lambda$-linear map

$$
\Psi_{\phi, \sigma}: Q H_{*}(M) \rightarrow Q H_{*+d}(M)
$$

as follows. For $a \in H_{*}(M, \mathbf{Z}), \Psi_{\phi, \sigma}(a)$ is the class in $Q H_{*+d}(M)$ whose intersection with $b \in H_{*}(M, \mathbf{Z})$ is given by:

$$
\Psi_{\phi, \sigma}(a) \cdot{ }_{M} b=\sum_{B \in \mathcal{H}} n(i(a), i(b) ; \sigma+i(B)) e^{B} .
$$

Here $n(v, w ; D)$ denotes the Gromov-Witten invariant which counts isolated $J$ holomorphic stable curves in $P_{\phi}$ of genus 0 and two marked points that represent the equivalence class $D$ and whose marked points go through given generic representatives of the classes $v$ and $w$ in $H_{*}\left(P_{\phi}, \mathbf{Z}\right)$. More precisely, one defines $n(v, w ; D)$ to be the intersection of the virtual moduli cycle

$$
\text { ev }: \overline{\mathcal{M}}_{0,2}^{\nu}\left(P_{\phi}, J, D\right) \rightarrow P_{\phi} \times P_{\phi},
$$

that consists of all perturbed $J$-holomorphic genus 0 stable maps that lie in class $D$ and have 2 marked points, with a generic representative of the class $v \otimes w$ in $P_{\phi} \times P_{\phi}$. This definition is well understood provided $M$ is spherically 
monotone or has minimal spherical Chern number $5 \geq n-1$. In the general case, the definition of Gromov-Witten invariants along these lines forms the subject of recent works $[\mathrm{FO}],[\mathrm{LiT}],[\mathrm{LT}],[\mathrm{R}]$ and $[\mathrm{HS}]$. Further, we have written $i$ for the homomorphism $H_{*}(M) \rightarrow H_{*}(P)$ and $\cdot_{M}$ for the linear extension to $Q H_{*}(M)$ of the usual intersection pairing on $H_{*}(M, \mathbf{Q})$. Thus $a \cdot{ }_{M} b=0$ unless $\operatorname{dim}(a)+\operatorname{dim}(b)=2 n$ in which case it is the algebraic number of intersection points of the cycles. Note finally that, by Gromov compactness, there are for each given energy level $\kappa$ only finitely many homology classes $D$ with $\omega(D-\sigma) \leq$ $\kappa$ that are represented by $J$-holomorphic curves in $P_{\phi}$. Thus $\Psi_{\phi, \sigma}(a)$ satisfies the finiteness condition for elements of $Q H_{*}(M, \Lambda)$.

Since $n(i(a), i(b) ; D)=0$ unless $2 c_{\phi}(D)+\operatorname{dim}(a)+\operatorname{dim}(b)=2 n$, we have

$$
\Psi_{\phi, \sigma}(a)=\sum a_{B} \otimes e^{B}
$$

where

$$
\operatorname{dim}\left(a_{B}\right)=\operatorname{dim}(a)+2 c_{\phi}(D)=\operatorname{dim}(a)+2 c_{\phi}(\sigma)+2 c(B) .
$$

Observe also that

$$
\Psi_{\phi, \sigma+B}=\Psi_{\phi, \sigma} \otimes e^{-B} .
$$

When $M$ is spherically monotone or has minimal spherical Chern number at least $n-1$ the following two results are proved by Seidel. The general case will be established in [LMP2].

Lemma 4.A. If $\phi$ is the constant loop $*$ and $\sigma_{0}$ is the flat section $p t \times S^{2}$ of $P_{*}=M \times S^{2}$ then $\Psi_{*, \sigma_{0}}$ is the identity map.

Proposition 4.B. Given sections $\sigma$ of $P_{\phi}$ and $\sigma^{\prime}$ of $P_{\psi}$ let $\sigma^{\prime} \# \sigma$ be the union of these sections in the fiber sum $P_{\psi} \# P_{\phi}=P_{\psi * \phi}$. Then

$$
\Psi_{\psi, \sigma^{\prime}} \circ \Psi_{\phi, \sigma}=\Psi_{\psi * \phi, \sigma^{\prime} \# \sigma} .
$$

The main step in the proof of these statements is to show that when calculating the Gromov-Witten invariant $n(i(a), i(b) ; D)$ via the intersection between the virtual moduli cycle and the class $i(a) \otimes i(b)$ we can assume the following: — the representative of $i(a) \otimes i(b)$ has the form $\alpha \times \beta$ where $\alpha, \beta$ are cycles lying in the fibers of $P_{\phi}$;

- the intersection occurs with elements in the top stratum of $\overline{\mathcal{M}}_{0,2}^{\nu}\left(P_{\phi}, J, D\right)$ consisting of sections of $P_{\phi}$.

Lemma $4 . \mathrm{A}$ is then almost immediate, and Proposition $4 . \mathrm{B}$ can be proved by the well-known gluing techniques of [RT] or [MS1].

\footnotetext{
5 The minimal spherical Chern number $N$ is the smallest positive integer such that the image of $c=c_{1}(T M)$ on $H_{2}^{S}(M)$ is contained in $N \mathbf{Z}$. It equals to $+\infty$ when this image vanishes.
} 
Corollary 4.C. $\Psi_{\phi, \sigma}$ is an isomorphism for all loops $\phi$ and sections $\sigma$.

With this in hand, we can establish Theorem 3.A and hence also 1.A.

Proof of 3.A:

Gromov-Witten invariants are linear in each variable. Thus if $i(a)=0$ for some $a \neq 0$, then $\Psi_{\phi, \sigma}(a)=0$, a contradiction with the fact that $\Psi_{\phi, \sigma}$ is an isomorphism.

\section{$\S 5$ The representation of $\pi_{1}(\operatorname{Ham}(M))$}

In this section we prove the following mild generalization of the main result in $[\mathrm{Se}]$.

Theorem 5.A. There exists a homomorphism

$$
\Psi: \pi_{1}(\operatorname{Ham}(M, \omega)) \rightarrow Q H_{\mathrm{ev}}\left(M, \Lambda_{R}\right)^{\times} .
$$

Our homomorphism is obtained from Seidel's by a process of averaging, and contains much the same information. In particular, his calculations show that it is nontrivial in many cases. Our averaging procedure forces us to work with the real Novikov ring $\Lambda_{R}$ which was introduced in the previous section. Note also that one cannot always replace $\Lambda_{R}$ by $\Lambda$ even when $\omega$ is integral unless $(M, \omega)$ is spherically monotone.

In order to use the maps $\Psi_{\phi, \sigma}$ to define a representation of the group $\pi_{1}(\operatorname{Ham}(M, \omega))$ we must make a canonical choice of section $\sigma_{\phi}$ that (up to equivalence) satisfies the composition rule

$$
\sigma_{\psi * \phi}=\sigma_{\phi} \# \sigma_{\psi}
$$

where $\sigma_{\phi} \# \sigma_{\psi}$ denotes the obvious union of the sections in $P_{\psi * \phi}=P_{\psi} \# P_{\phi}$. Unfortunately, it is not always possible to do this if one just considers usual sections. Further, one has to proceed slightly differently in the case when the classes $[\omega]$ and $c=c_{1}(T M)$ are linearly dependent on $H_{2}^{S}(M)$. So let us assume to begin with that these classes are linearly independent.

We will say that $\sigma$ is an $\mathbf{R}$-section of $P_{\phi}$ if it is a finite $\operatorname{sum} \sum \lambda_{i} \sigma_{i}, \lambda_{i} \in \mathbf{R}$, of sections such that $\sum \lambda_{i}=1$. Then, by our assumption on $[\omega]$ and $c$, there is an $\mathbf{R}$-section $\sigma_{\phi}$ such that

$$
u_{\phi}\left(\sigma_{\phi}\right)=0, \quad c_{\phi}\left(\sigma_{\phi}\right)=0 .
$$

Clearly, the equivalence class of this $\mathbf{R}$-section is unique. Also, by Lemma 3.E the needed composition rule holds. Further, the definition of the map $\Psi_{\phi, \sigma_{\phi}}$ still makes perfect sense provided that one allows the coefficients $B$ to belong

\footnotetext{
${ }^{6}$ In fact, when $(M, \omega)$ is spherically monotone, we may take the range of this homomorphism to be $Q H_{\mathrm{ev}}(M, \Lambda)^{\times}$. This case of our theorem was proved in the first version of Seidel's paper.
} 
to $\mathcal{H}_{R}=H_{2}^{S}(M, \mathbf{R}) / \sim$ so that the sum $\sigma_{\phi}+B$ can be integral. We therefore get a representation

$$
\rho: \pi_{1}(\operatorname{Ham}(M, \omega)) \rightarrow \operatorname{Hom}_{\Lambda_{R}}\left(Q H_{*}\left(M, \Lambda_{R}\right)\right)
$$

of $\pi_{1}(\operatorname{Ham}(M))$ in the group of automorphisms of the $\Lambda_{R}$-module $Q H_{*}\left(M, \Lambda_{R}\right)$.

One should think of the $\mathbf{R}$-section $\sigma_{\phi}$ as an average of the sections in $P_{\phi}$. The effect of enlarging the Novikov ring to $\Lambda_{R}$ is thus to make enough room to take this average.

Now consider the case when the classes $[\omega]$ and $c$ are linearly dependent on $H_{2}^{S}(M)$. The difficulty here is that the canonical extensions $u_{\phi}$ and $c_{\phi}$ need not be dependent on $H_{2}^{S}\left(P_{\phi}\right)$. (For example, consider the case when $\phi$ is a rotation of $M=S^{2}$.) Therefore, there may be no $\mathbf{R}$-section such that $u_{\phi}\left(\sigma_{\phi}\right)=0$, $c_{\phi}\left(\sigma_{\phi}\right)=0$. However, in this case, the equivalence relation on $\mathcal{H}_{R}$ is given simply by $[\omega]$. Moreover, if the class $u_{\phi}$ has the same value on the two sections $\sigma, \sigma^{\prime}$, so does $c_{\phi}$. Hence it suffices to choose $\sigma_{\phi}$ so that $u_{\phi}\left(\sigma_{\phi}\right)=0$. The value of $c_{\phi}$ on $\sigma_{\phi}$ is the same for all choices of $\sigma_{\phi}$ (though it may not be zero), and so $\sigma_{\phi}$ is still unique up to equivalence. Thus the previous arguments go through.

In order to complete the proof of Theorem 5.A we have to show that the automorphisms in the image of $\rho$ commute with the quantum intersection product. To do this, it is useful to describe the homomorphism $\rho$ in the terms used by Seidel. Recall that the quantum intersection product $a *_{M} b$ of two classes $a \in H_{i}(M, \mathbf{Q}), b \in H_{j}(M)$ is defined as follows:

$$
a *_{M} b=\sum_{B \in \mathcal{H}}\left(a *_{M} b\right)_{B} \otimes e^{B},
$$

where $\left(a *_{M} b\right)_{B} \in H_{i+j-2 n+2 c(A)}$ is defined by the requirement that

$$
\left(a *_{M} b\right)_{B} \cdot{ }_{M} c=n_{M}(a, b, c ; B),
$$

where $n_{M}(a, b, c ; B)$ is the "number of isolated $J$-holomorphic spheres in class $B$ that meet $a, b$, and $c$ ". More precisely, $n_{M}(a, b, c ; B)$ is the Gromov-Witten invariant that counts the number of (perturbed) $J$-holomorphic curves in class $B$ that meet the classes $a, b$ and $c$. This product is extended to $Q H_{*}(M)$ by linearity over $\Lambda$. It clearly extends also to $\Lambda_{R}$. Note here that when defining $a *_{M} b$ we still sum over classes $B \in \mathcal{H}$ (and not $B \in \mathcal{H}_{R}$ ), since $J$-holomorphic spheres can only represent integral classes.

The next proposition appears in Seidel when $M$ satisfies the semi-positivity condition described in $\S 6$. The proof in general follows by looking at what happens to the Gromov-Witten invariants $n(i(a), i(b), D)$ when the representatives of $i(a)$ and $i(b)$ are taken to lie in the same fiber.

Proposition 5.B. For all $\phi \in \pi_{1}(\operatorname{Ham}(M))$,

$$
\rho(\phi)(a)=\Psi_{\phi, \sigma_{\phi}}([M]) *_{M} a .
$$




\section{Proof of Theorem 5.A.}

This is now clear. By Proposition 5.B the homomorphism

$$
\Psi: \quad \pi_{1}(\operatorname{Ham}(M)) \rightarrow Q H_{*}\left(M ; \Lambda_{R}\right)^{\times}
$$

is given by $\phi \mapsto \rho(\phi)([M])$.

\section{$\S 6$ What is proved and what will be proved}

The outline of the proof of Theorem 1.A (or of the equivalent Theorem 3.A) given above becomes completely rigorous provided $(M, \omega)$ satisfies one of the following assumptions which were used by Seidel in [Se] for definition of his action:

(i) $(M, \omega)$ is spherically monotone;

(ii) The minimal spherical Chern number $N$ does not exceed $n-1$. The same is true for our results in $\S 5$.

The reduction of our results in section 2 and 3 to 1.A is "soft" and works without any additional conditions. The only point where one should be careful is that we need 1.A to hold simultaneously for all symplectic forms under consideration. In view of this, 2.A is proved provided each of two symplectic forms satisfy either (i) or (ii), while for 2.B and 3.B one needs to assume the deformation invariant assumption (ii).

In [LMP2] we will prove 1.A in full generality, and thus all the results of the present paper will be confirmed without additional assumptions.

Acknowledgment. We thank Paul Seidel for stimulating discussions and for useful critical remarks on a preliminary version of this paper. We are also grateful to Dietmar Salamon for helpful discussions.

\section{References}

[BP] M. Bialy and L. Polterovich, Hamiltonian diffeomorphisms and Lagrangian distributions, Geometric and Funct. Analysis, 2 (1992), 173-210.

[FO] K. Fukaya and K. Ono, Arnold conjecture and Gromov-Witten invariants, preprint (1996)

[G] R. Gompf, A new construction for symplectic 4-manifolds, Annals of Mathematics, 142 (1995), 527-595.

[GH] P. Griffiths and J. Harris, Principles of algebraic geometry. Wiley, New York (1978).

[HS] H. Hofer and D. Salamon, in preparation.

$[\mathrm{K}]$ F. Kirwan, Cohomology of quotients in symplectic and algebraic geometry. Mathematics Notes, 31. Princeton University Press (1984). 
[LMP1] F. Lalonde, D. McDuff and L. Polterovich, On the Flux conjectures, preprint dg-ga/9706015, to appear in the Proceedings of the CRM Workshop on Geometry, Topology and Dynamics, Montreal 1995, CRM Special Series pubished by the AMS, 1997.

[LMP2] F. Lalonde, D. McDuff and L. Polterovich, in preparation.

[LiT] Jun Li and G. Tian, Virtual moduli cycles and Gromov-Witten invariants of general symplectic manifolds, preprint (1996)

[LT] G. Liu and G. Tian, Floer homology and Arnold conjecture, preprint (1996)

[LO] G. Lupton and J. Oprea, Cohomologically symplectic spaces, Toral actions and the Gottlieb group. Preprint.

[M1] D. McDuff, Symplectic diffeomorphisms and the flux homomorphism, Inventiones Mathematicae, 77, (1984) 353-66.

[M2] D. McDuff, Recent developments in Symplectic Topology, to appear in Proceedings of the 2nd European Congress, Budapest (1996)

[MS1] D. McDuff and D.A. Salamon, J-holomorphic curves and quantum cohomology, Amer Math Soc Lecture Notes \#6, Amer. Math. Soc. Providence (1995).

[MS2] D. McDuff and D.A. Salamon, Introduction to Symplectic Topology, OUP, Oxford, (1995)

[PSS] S. Piunikhin, D. Salamon and M. Schwarz, Symplectic Floer-Donaldson theory and Quantum Cohomology, Contact and Symplectic Geometry ed C. Thomas, Proceedings of the 1994 Newton Institute Conference, CUP, Cambridge (1996)

[P1] L. Polterovich, Hamiltonian Loops and Arnold's principle, preprint (1996), to appear in the volume in honor of V.I. Arnold.

[P2] L. Polterovich, Symplectic aspects of the first eigenvalue, preprint dgga/9705003.

[R] Y. Ruan, Virtual neighbourhoods and pseudo-holomorphic curves, Preprint alg-geom/9611021

[RT] Y. Ruan and G. Tian, A mathematical theory of quantum cohomology. Journ Diff Geo 42 (1995), 259-367.

[Se] P. Seidel, $\pi_{1}$ of symplectic automorphism groups and invertibles in quantum cohomology rings, preprint dg-ga/9511011, (1995) revised (1997), to appear in Geom. and Funct. Anal. 